TAO, Vol. 12, No. 1, 137-154, March 2001

\title{
Numerical Simulation of Contrail Microphysical and Radiative Properties
}

\author{
Jen-Ping Chen ${ }^{1}$ and Ruei-Fong $\operatorname{Lin}^{2, *}$
}

(Manuscript received 3 January 2001, in final form 22 February 2001)

\begin{abstract}
The late-dispersion phase of contrails is simulated with a high-resolution cloud model in which the size distributions of ice crystals are explicitly computed. The cloud optical properties derived from this model are then used to compute the cloud reflectivity using the Monte Carlo method for the shortwave radiation, as well as the outgoing longwave radiative fluxes at the top of atmosphere using the absorption approximation method. Simulations for both the dissipating and persistent contrail scenarios in typical ambient conditions over Taiwan showed that the evolution of contrail morphology is critically controlled by the ambient humidity. Also, contrails may effectively alter the local radiative budget, more so for the persistent contrails than the dissipating contrails.
\end{abstract}

(Key words: Contrail, Microphysics, Radiative forcing)

\section{INTRODUCTION}

Many studies indicate that the global occurrence of contrails, formed by mixing of the hot and moist aircraft exhaust with the cold ambient air, has been increasing with the growth of the commercial aircraft traffic (e.g., Changnon 1981; Seaver and Lee 1987; Frankel et al. 1997; Boucher 1999; Minnis et al. 1999). Studies of the high and thin natural cirrus show that the net radiative forcing by this cloud genre could be either positive or negative depending sensitively on their height and optical properties and a few external parameters, such as the solar zenith angle, surface albedo, and the thermodynamic profile of the air column (Ackerman et al. 1988; Stephens et al. 1990). Cirrus enhances outgoing shortwave (SW) radiation by reflecting the incident solar radiation. Yet, this negative SW radiative forcing is partially compensated or in certain conditions even overwhelmed by the reduction of outgoing longwave (LW) radiation

\footnotetext{
${ }^{1}$ Department of Atmospheric Sciences, National Taiwan University, Taipei, Taiwan, ROC

${ }^{2}$ Currently at the Goddard Earth Sciences and Technology Center, University of Maryland, Baltimore County, USA

${ }^{*}$ Corresponding Author Address: Dr. Ruei-Fong Lin, Goddard Earth Sciences and Technology Center, University of Maryland, Baltimore County, USA; E-mail: lin@climate.gsfc.nasa.gov
} 
by the cloud. This is because such high clouds may effectively absorb upward terrestrial radiation emitted by the Earth's surface and the air column beneath the cloud, yet concurrently, it emits at a temperature far less than the Earth's surface and thus reduces the outgoing LW radiation. All these radiative effects are highly controlled by the microphysical properties of these cirrus clouds.

The microphysical properties of contrails in their early stage differ distinctly from those of natural cirrus. The sizes of ice crystals in contrails appear to be much smaller than in the natural cirrus, whereas the number density of ice crystals in contrails is much higher (Goodman et al. 1998). Yet, contrails may evolve to possess similar optical properties as the surrounding cirrus. As pointed out by Minnis et al. (1998), aged contrails often cannot be distinguished from natural cirrus clouds. So, the microphysical and radiative properties of contrails can vary significantly not only with ambient conditions but also with time.

Assessment of contrail radiative forcing is a challenging task because of the uncertainties regarding cloud coverage and cloud properties. There have been quite a few sensitivity studies on the radiation budget of contrails done with assumed cloud ice water path (IWP), effective radius and external parameters (e.g., Fortuin et al. 1995; Meerkotter et al. 1999). In a rather extensive investigation, Fortuin et al. (1995) estimated the net radiative forcing by contrails to be $-0.15 \sim 0.3 \mathrm{~W} \mathrm{~m}^{-2}$ in summer and $0.05 \sim 0.3 \mathrm{~W} \mathrm{~m}^{-2}$ in winter for the North Atlantic flight forridors. They estimated the radiative forcing of contrails by using effective radii ranging from 4 to $100 \mu \mathrm{m}$ and IWP ranging from 4 to $50 \mathrm{~g} \mathrm{~m}^{-2}$. The negative net radiative forcing occurs in the summer time for contrails with small ice crystals and high IWP. These results demonstrated the intricate sensitivity of contrail radiative forcing to the assumed internal and external parameters. Therefore, the effects of contrails on the regional radiation budget should be better estimated by using external parameters pertaining to the specific region.

Furthermore, many studies similar to Fortuin et al.'s (1995) treated contrails as plane parallel and vertically homogeneous cirrus clouds. This assumption is often thought of as secondary significance compared to other internal and external parameters discussed in the above paragraph. However, Schulz (1998) pointed out that differences in shortwave (SW) cloud reflectivity between homogeneous and inhomogeneous clouds increase with contrail optical depth. Cloud inhomogeneity is important if the cloud is thick enough. It is thus of interest to check the effects of cloud inhomogeneity on the computation of radiation fields over and under a contrail, particularly the persistent ones. In order to achieve this goal, spatial distribution of ice particles in contrails has to be simulated. As pointed out by Gieren (1996) and Chlond (1998), large eddy simulation (LES) is more suitable than regional models in simulating the evolution of contrail microphysical structures due to the particular spatial dimensions of these clouds. Uncertainty in the estimate of contrail radiative forcing in the regional and global scales may be reduced using the output of such fine-resolution models. With the LES, effects of cloud inhomogeneity can be evaluated either by Monte Carlo method (MCM) or the individual pixel approximation (IPA), which will be discussed further later.

This paper is the first part of a series of detailed investigations on contrail radiation budget over the Taiwan area, with foci on the microphysical and radiative properties of evolving contrails. We adopted the LES approach and compared radiation field computed either by the MCM or by IPA. Our model was then used to produce contrail properties for simulating the 
contrail effect on regional radiation budgets in Chen et al. (2001) and Wang et al. (2001).

\section{MODEL DESCRIPTION}

In this study we adopted a cloud-resolving model of Lin (1997), which is a two-dimensional model with explicit calculation of binned ice crystal size distribution. The crystal size distribution is allowed to evolve by vapor-diffusion growth, advection by cloud scale and subgrid-scale turbulences, and gravitational sedimentation. This model has been used to study cirrus outflow dynamics (Lin 1997) and sustainability of high tropical cirrus (Boehm et al. 1999). Furthermore, this model and the parcel version of it performed competitively in the Idealized Cirrus Model Comparison Project (Starr et al. 2000) and the Cirrus Parcel Model Comparison Project (Lin et al. 2000), respectively. Some modification was made in order to simulate the contrails.

It is assumed that the model domain represents a cross-section of the contrail. The width and the height of the model domain are 1.5 and $1.0 \mathrm{~km}$, respectively, with a grid resolution of $25 \mathrm{~m}$ in both directions. The framework of the dynamics module is similar to the cloudresolving model of Starr and Cox (1985). The major improvements are: (1) the use of Smagorinsky-Lilly-type parameterization for eddy coefficients (Lilly 1962); (2) the implementation of artificial sponge layers to reduce gravity wave reflection; (3) the incorporation of a high-resolution, positive-definite scheme for calculating particle ransport (Hirsch 1988); and (4) the use of the Adams-Bashforth scheme as the temporal integration scheme. The prognostic variables of the models are vorticity, potential temperature, water vapor mixing ratio, ice crystal number density and mean ice mass in each bin; whereas the stream function is diagnosed. All calculations are done with the assumption of incompressible fluid and Bussinesq approximation.

An initially monodispersed size distribution of ice crystals is assumed throughout the original cloudy area. All ice crystals are assumed to be spherical with a bulk density of $0.9 \mathrm{~g}$ $\mathrm{cm}^{-3}$, distributed over 25 bins with corresponding sizes ranging from 1 to $100 \mu \mathrm{m}$. Note that no ice crystals become larger than the bin limit $100 \mu \mathrm{m}$ within the time span of our simulations. Computation of the evolution of ice crystal size distributions due to diffusional growth follows the modified-Young-type scheme (Young 1974; Chen and Lamb 1994), in which the ice crystal number concentration and mass are conserved during the partitioning and redistribution into different bins. Aggregation is found of no importance in a sensitivity test and is thus switched off in all of the simulations reported in this study. The ice supersaturation-dependent heterogeneous nucleation parameterization (Meyers et al. 1992) is switched off due to insignificant number of ice nuclei in the high altitudes where contrails form; whereas the homogeneous freezing of haze particles is never triggered after the late-dispersion phase of contrail formation. This means that no new ice particles are formed in our simulations that focused on the late-dispersion phase, and unsurprisingly the domain-integrated ice particle number concentration will decrease with time because of fall out and, in the case of dissipating contrail, complete evaporation.

Chlond (1998) reported that the evolution of contrail is not sensitive to the radiation 
processes. If one may assume that radiative warming decreases linearly within contrail, then buoyancy production due to radiative destabilization would be proportional to the differential radiative warming and the depth of the layer (Lilly 1988). In fact, the depth of a young persistent contrail is only of several hundred meters, as shown in the later section. On top of that, ice crystals are concentrated within the upper $100 \mathrm{~m}$ of the persistent contrails at $30 \mathrm{~min}$ of simulation time. Therefore, the effective depth to generate radiatively-driven turbulent energy is small. As a result, the secondary circulation within and around the contrail is driven mainly by the diabatic cooling (warming) due to ice sublimation (vapor deposition). Thus, the interactive radiation was switched off in the simulations in order to reduce the simulation time. Radiation, however, may play a more significant role in contrail evolution beyond the 30 minute period of time.

\section{RADIATIVE FORCING ESTIMATE}

Given the ice crystal size distribution in a grid box, the shortwave optical properties are computed following Ebert and Curry (1992). The longwave volume absorption coefficient $\left(b_{a b s}\right)$ is obtained directly through

$$
b_{a b s}=\sum_{i} Q_{a b s, i} \cdot r_{i}^{2} \cdot N_{i}
$$

where $Q_{a b s}$ is the absorption efficiency from Mie theory, $r$ is the particle radius, $N$ is the particle number concentration, and the subscript $i$ denotes the bin number.

Most schemes used to parameterize the optical properties of ice clouds (e.g., Ebert and Curry 1992; Fu and Liou 1993) are designed for natural cirrus, in which the effective radius is usually larger than $10 \mu \mathrm{m}$. Direct extrapolation of these formulae may lead to an overestimate of the absorption coefficient of small particles at IR wavelengths. As shown in Fig. 1, the ratio of extinction efficiency to absorption efficiency $\left(Q_{e x, S W} / Q_{a b s, L W}\right)$ is $\sim 2$ for large particles, but increases sharply as particle radius becomes increasingly smaller than $5 \mu \mathrm{m}$. Given two clouds of the same SW optical depth, the cloud with small ice particles $(r<5 \mu \mathrm{m})$ would have much smaller LW radiative forcing than one containing larger ice particles $(r>10 \mu \mathrm{m})$. Therefore, the threshold optical depth, at which the net radiative forcing is zero, of a cloud containing small ice particles would be much lower than that containing large ones.

Longwave radiative transfer is calculated here using the absorption approximation method in which the Planck function is approximated as a linear function of the optical depth (Toon et al. 1989). By applying the Individual Pixel Approximation in the longwave flux calculation, it is further assumed that radiative transfer through each column is not affected by the adjacent columns. The LW routine of this model is tested to produce comparable outgoing longwave radiative flux in the mid-latitude summer high cloud situation (Fu et al. 1997). However, ignoring infrared scattering by ice particles will result in an underestimated top-of-atmosphere (TOA) outgoing longwave flux. As indicated by Fu et al. (1997), the bias was $6 \mathrm{~W} \mathrm{~m}^{-2}$ for high cirrus cloud $\left(\tau_{\mathrm{sw}}=0.8\right.$ ) under mid-latitude summer condition.

The reflectivity of the contrail with respect to the direct solar radiation is estimated with 


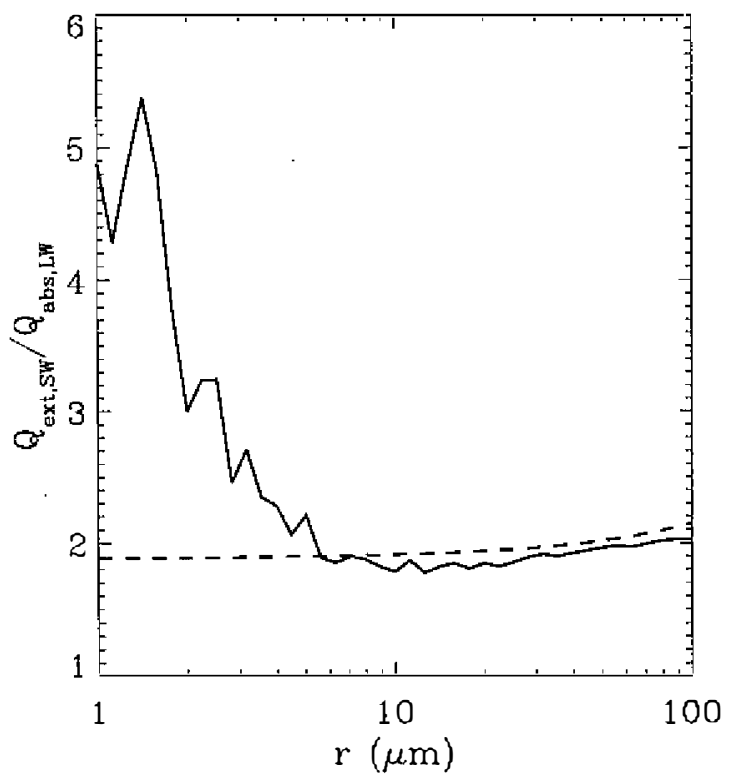

Fig. 1. The ratio of the extinction efficiency at $0.55 \mu \mathrm{m}$ over the absorption efficiency at $11 \mu \mathrm{m}$ as a function of particle radius (the solid curve). These two parameters are computed using a standard Mie scattering code. Also shown is the same ratio but derived from Ebert and Curry's parameterization scheme (the dashed curve).

the Monte Carlo method (e.g., Danielson et al. 1969; Davies 1978; Chylek and Dobbie 1995). The commonly used plane-parallel approximation in the computation of radiation fields over a cloud with finite geometry does not account for radiation that diffuses out through the cloud sides as well as the illumination of the cloud side by direct solar radiation (Davies 1978). The Monte Carlo method used here, on the other hand, can compute the radiation field over a cloud with complicated geometry and spatial inhomogeneity. Scattering of light by hydrometeors is viewed as collisions of photons onto hydrometeors. These collisions satisfy the Poisson process (e.g., Walpole and Myers 1990). So, here we have the extinction coefficient, single scattering albedo, and phase function as the controlling factors of the probability distribution functions (PDF) for scattering. In a homogeneous medium, the PDF of the traveling distance $L$ between two collisions is a function of the mean free path $l_{0}=1 / b_{e x t}$, and is formulated as

$$
P(L)=\frac{1}{l_{0}} \exp \left(\frac{L}{l_{0}}\right) .
$$

We can then define a dimensionless parameter $x=L / l_{0}$, the PDF of $x$ becomes

$$
P(x)=\exp (-x)
$$

In a medium of spatially varying $b_{e x t}$, Eq. (3) is valid when transforming the physical space into an optical-path space. The parameter $x$ can be discretized as

$$
x=\sum_{k} \frac{\Delta d_{k}}{b_{e x t, k}},
$$

where $k$ is the grid box index along the photon path and $\Delta \boldsymbol{d}_{k}$ is length of the segment of photon 
path within grid box $k$ (see Fig. 2). So, after $x$ is acquired, the actual distance between two collisions can be formulated as (Chylek and Dobbie 1995):

$$
L=\sum_{k} \Delta d_{k}
$$

Given the single scattering albedo, the probability that a photon would be absorbed by a hydrometeor at one collision is specified. Then the PDF of the photon scattering angle with respect to a given incident angle can be obtained from the phase function, which is further discussed below.

It has been noted that neither the equivalent-surface sphere nor the equivalent-volume sphere assumptions could properly estimate the SW optical and radiative properties of clouds that contain non-spherical particles (Kinne and Liou 1989). Therefore, the phase functions of spherical and non-spherical particles can be quite different. However, for the sake of consistency, the Henyey-Greenstein phase function

$$
P(\cos \Theta)=\left(1-g^{2}\right) /\left(1+g^{2}-2 g \cos \Theta\right)
$$

was adopted in this study since we assumed spherical ice crystals in the microphysical module. In the above equation, $\Theta$ is the scattering angle relative to the direction of the incoming photon, and $g$ is the asymmetry factor. Photon trajectories can thus be simulated as randomwalk processes, of which the distance between two turns, and the direction of the turn are based on the PDFs. In this way, the reflectivity and transmissivity are computed as the number fraction of the incident photons that leave the contrail with a zenith angle greater and smaller than $90^{\circ}$, respectively (downward irradiance with zenith angle between $0^{\circ}$ and $90^{\circ}$; upward irradiance between $90^{\circ}$ and $180^{\circ}$ ). Note that the total number of the input incident photons has to be big enough to render a statistically meaningful reflectivity, as was done in this study.

\section{SIMULATIONS}

The wake of aircraft exhaust goes through jet, vortex, dispersion, and diffusion regimes. Our simulations are assumed to start around $1000 \mathrm{~s}$ after the exhaust plume exits the engine

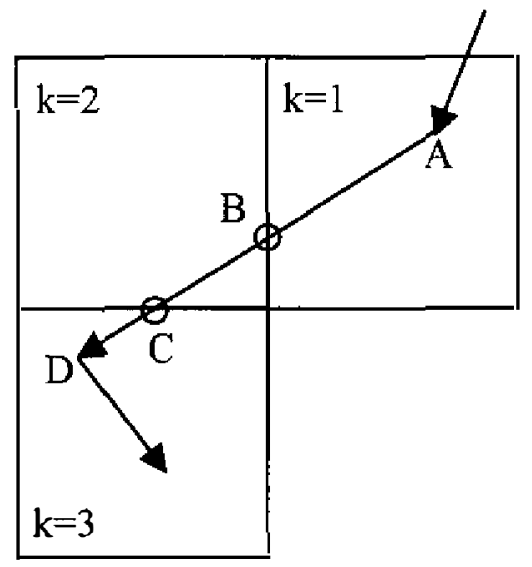

Fig. 2. A schematic of a segment of a photon trajectory. The points A and D indicate the locations of two consecutive collisions, whereas $\mathrm{B}$ and $\mathrm{C}$ indicate the points (empty circles) where the photon went across the grid boundaries. In this case, the free path

$$
L=\frac{\overline{A B}}{b_{e x t, 1}}+\frac{\overline{B C}}{b_{e x t, 2}}+\frac{\overline{C D}}{b_{e x t, 3}} \text {. }
$$


nozzle, which corresponds to the beginning of the diffusion regimes. At this regime, the atmospheric conditions totally control the further dispersion of the exhaust plume. For the simulations shown below, we use the average pressure $(260 \mathrm{hPa})$, temperature $\left(-49.7^{\circ} \mathrm{C}\right)$ and lapse rate $\left(-6.91^{\circ} \mathrm{C} \mathrm{km}^{-1}\right)$ at $10.5 \mathrm{~km}$ as the environmental conditions according to the BanChao sounding data of the winter of 1997. Initial conditions for the plume are similar to those used in Chlond (1998), as described below.

The model domain centers at $10.5 \mathrm{~km}$ above the sea level. The initial contrail is assumed to be $200 \mathrm{~m}$ in width and $150 \mathrm{~m}$ in depth. The temperature inside the cloud is assumed to be ice moist adiabatic. The initial ice crystal number concentration is $320 \mathrm{~cm}^{-3}$. It is further assumed that ice crystals are monodispersed with a radius of $2.2 \mu \mathrm{m}$ according to Chlond (1998). Therefore, the initial ice mixing ratio is about $17 \mathrm{mg} \mathrm{cm}^{-3}$. The relatively warm plume is assumed to rise in a motionless, hydrostatic and stratified environment.

A small initial perturbation (e.g., $10^{-3} \mathrm{~K}$ in Chlond 1998), as is frequently used in LES of the planetary boundary layer, extends the model-spinning time and entails a better model response. However, this approach may not be suitable for a 30-min simulation because eddies are not yet fully developed within this time frame. We chose to randomly perturb potential temperature in the initial contrail region with an amplitude of $0.1 \mathrm{~K}$, which is of the scale of cirrus turbulence (Starr and Cox 1985). The differences in the initial perturbation between this work and Chlond (1998) result in differences mainly in the development of wind fields. We acknowledge that a better choice might be to initiate the model microphysical and dynamic fields using output of a jet-vortex-phase-resolving model.

Only two sets of detailed results, each corresponds to the dissipating and persistent contails, are discussed in this study (Table 1). As shown in Chen et al. (2001), the ambient relative humidity RHi is less than $100 \%$ in the dissipating contrail simulation (Run 1) and greater than $100 \%$ in the persistent contrail simulation (Run 2). The ambient conditions satisfy the SchmidtAppleman criterion for contrail formation (Schumann 1996; Chen et al. 2001).

Table 1. The simulation set.

\begin{tabular}{|c|c|c|}
\hline Identifier & Description & Ambient $\mathrm{RH}_{\mathrm{i}}(\%)$ \\
\hline Run 1 & dissipating & 80 \\
\hline Run 2 & persistent & 120 \\
\hline
\end{tabular}

In the beginning of the simulation, the cloudy region rises because it is slightly warmer than the environment (Fig. 3). This rising motion induces clockwise and counterclockwise circulations at the right and left cloud boundaries, respectively. Ambient air is then entrained into the edges of the contrails, followed by the development of secondary circulation at the lower right and left flanks of the cloud. As pointed out by Chlond (1998), the vertical extent of the turbulent region is mainly controlled by the static stability of the ambient layer. Therefore, as time proceeds, the turbulent region gradually flattens because the prescribed environment is 

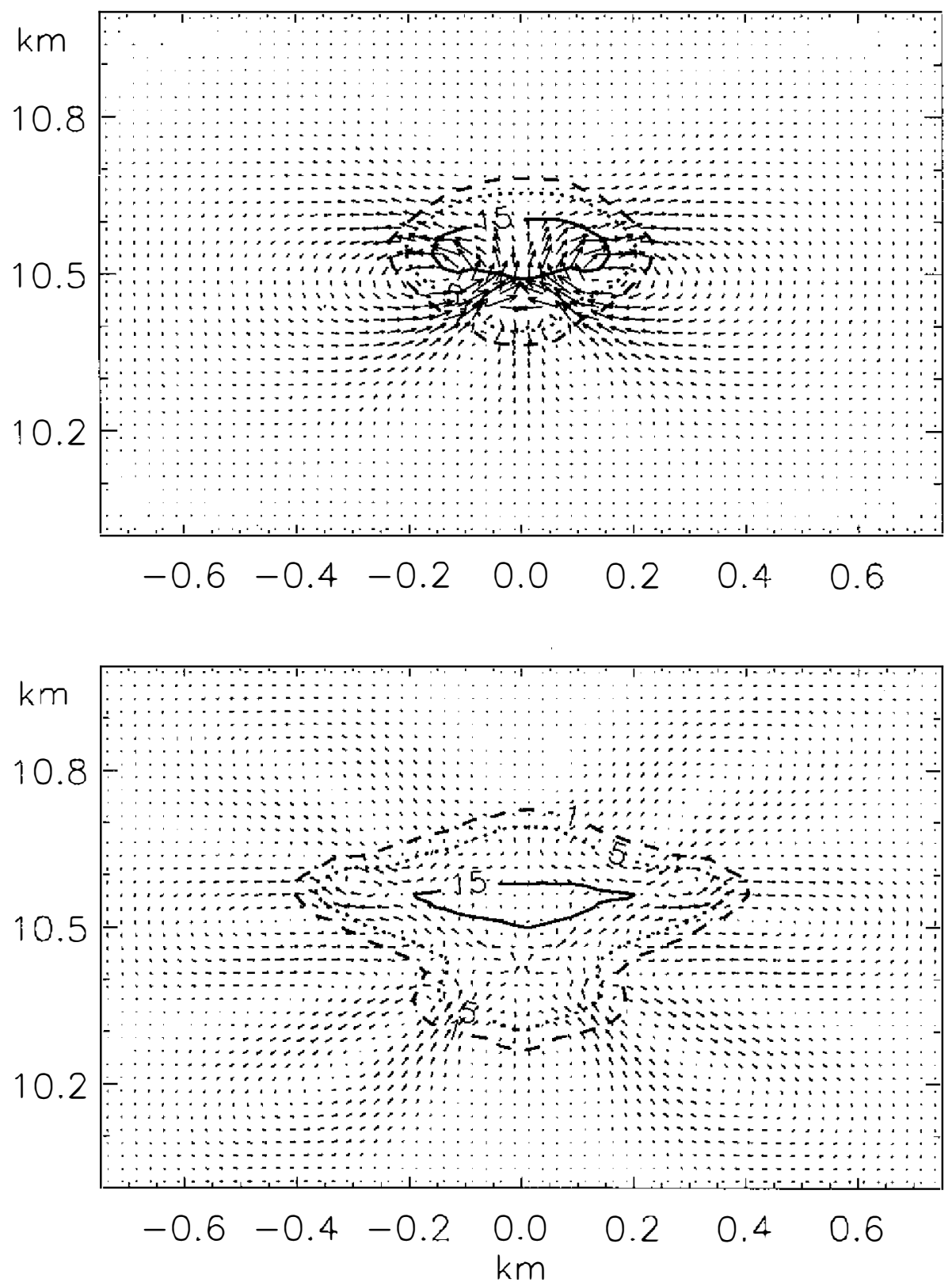

Fig. 3. Velocity vectors of the resolved-scale velocity field of the persistent contrail at $5 \mathrm{~min}$ (the upper panel) and $15 \mathrm{~min}$ (the lower panel) of simulation time. Superimposed are contours of IWC of 1,5 and $15 \mathrm{mg} \mathrm{m}^{-3}$; (dashed, dotted, and solid curves, respectively). Wind vectors with a length of one grid cell indicate wind speed $0.2 \mathrm{~m} \mathrm{~s}^{-1}$. 
static stable. The evolution of contrail morphology shown in Fig. 3 is in reasonably good agreement with the simulations of Chlond (1998). The wind field in this study evolves differently due to the differences in the amplitude of initial perturbation, as discussed in the previous section. The discrepancies could also have resulted from the inherent disparity between the 2$\mathrm{D}$ and 3-D framework, as pointed out by Chlond (1998). The 2-D and 3-D turbulence is fundamentally different because the former does not include vortex stretching. Furthermore, energy cascading differs in 2-D and 3-D turbulence. The differences in the subgrid-scale parameterization between Chlond (1998) and this study may also affect the dissipation rate of turbulent kinetic energy as well as the contrail-spreading rate.

In the dissipating contrail scenario (Run 1), mixing of the subsaturated air into the cloudy air causes the evaporation of ice crystals that entails a gradual shrinking of the contrail (Fig. 4a). In the persistent contrail scenario (Run 2), the cloudy region expands horizontally (Fig. 5a), mainly because the entrainment and sub-grid scale diffusion of moist air results in the growth of ice particles. The vertical extent of the cloudy region expands downward due to sedimentation of large ice crystals.

Run 1 and 2 are contrasting examples showing that the evolution of contrail morphology is critically controlled by the ambient humidity. Whether the air is saturated (with respect to ice) or not is the principal criterion that determines the fate (growth or decay) of the contrails. Furthermore, as shown by Chen et al. (2001; Table 2), the microphysical and radiative characteristics of the same type of contrails (persistent or dissipating) also vary significantly with different ambient relative humidities.

Ice particle concentration and ice water content are highly peaked between the heights 10.5 and $10.6 \mathrm{~km}$ throughout the entire $30 \mathrm{~min}$ of simulation in both cases. After $30 \mathrm{~min}$, IWC in the core region decreases to less than $10 \mathrm{mg} \mathrm{cm}^{-3}$ in the dissipating contrail (Fig. 4a), but remains around $16 \mathrm{mg} \mathrm{cm}^{-3}$ in the persistent contrail (Fig. 5a). The ice particle number concentration remains above $100 \mathrm{~cm}^{-3}$ in the core of both types of contrails. Such number concentrations are much higher than those in most natural cirrus, except perhaps in tropical cirrus anvil where ice crystal number concentration of $1 \mathrm{~cm}^{-3}$ may be found (Knollenberg et al. 1993; Chen et al. 1997). A significant contrast exists between the effective radii of these two contrails. As shown in Fig. 4c, the effective radii remain less than $3 \mu \mathrm{m}$ in the dissipating contrail. In the persistent contrail (Fig. 5c) the effective radii are still less than $3 \mu \mathrm{m}$ in the core, but become increasingly large toward the cloud edges, particularly at the lower part of the contrail. Such a phenomenon can be better understood with the evolution of particle size distribution as will be discussed later.

Figures $4 \mathrm{~d}$ and $5 \mathrm{~d}$ show the vertically integrated optical properties, including ice water path, SW optical depth at wavelength between 0.3 and $0.7 \mu \mathrm{m}$, LW optical depth at wavelength of $10.75 \mu \mathrm{m}$, and cloud reflectivity at incident solar zenith angle $\mu_{0} \equiv \cos \Theta_{0}=0.6$ computed using IPA. All these values are higher near the center of the contrails, and are higher in the persistent contrail than in the dissipating contrail. The maximum ice water path is less than $1 \mathrm{~g} \mathrm{~m}^{-2}$ in the dissipating contrail, but is five times as large in the persistent contrail. The SW optical depths are more than twice of the LW optical depths for both contrail types. We will show later that such a big difference between the SW and LW optical depths has a significant impact on the sign of net radiative forcing. The maximum SW optical depth is 


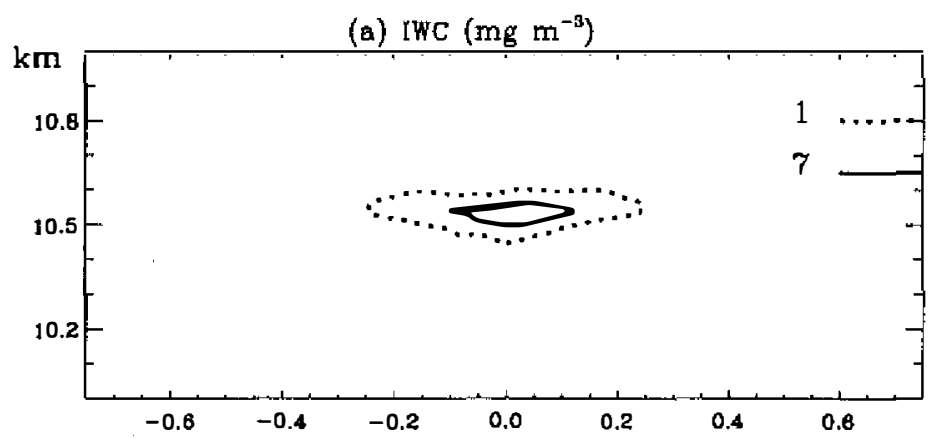

(b) $\mathrm{N}_{1}\left(\mathrm{~cm}^{-3}\right)$

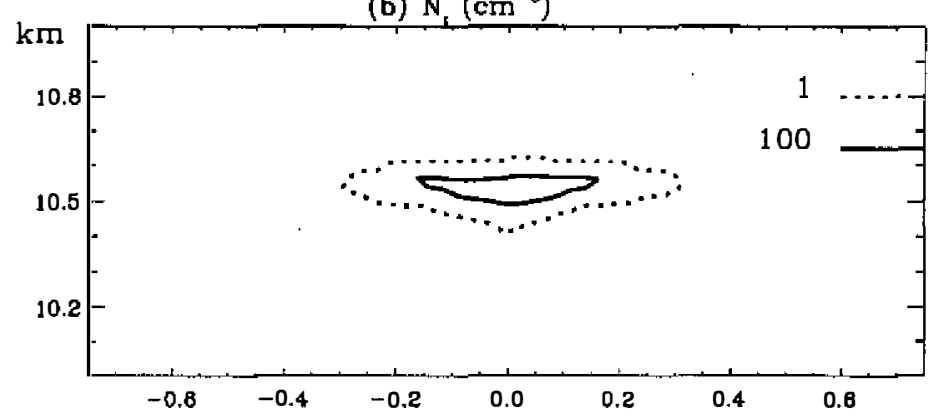

(c) $r(\mu m)$

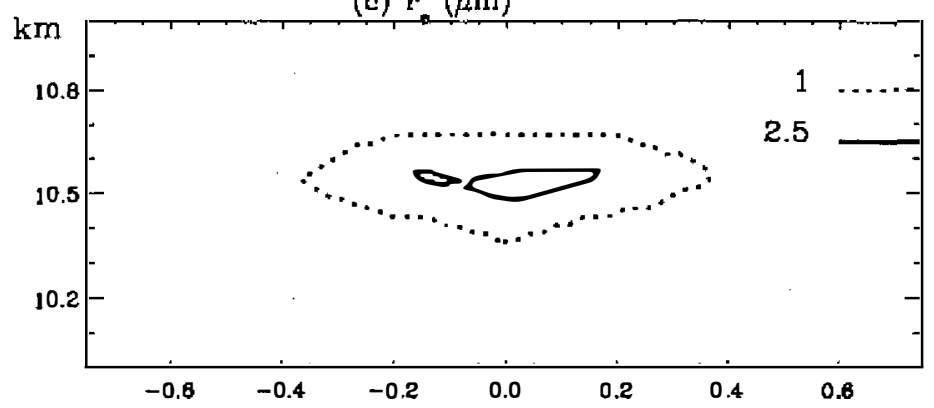

(d)

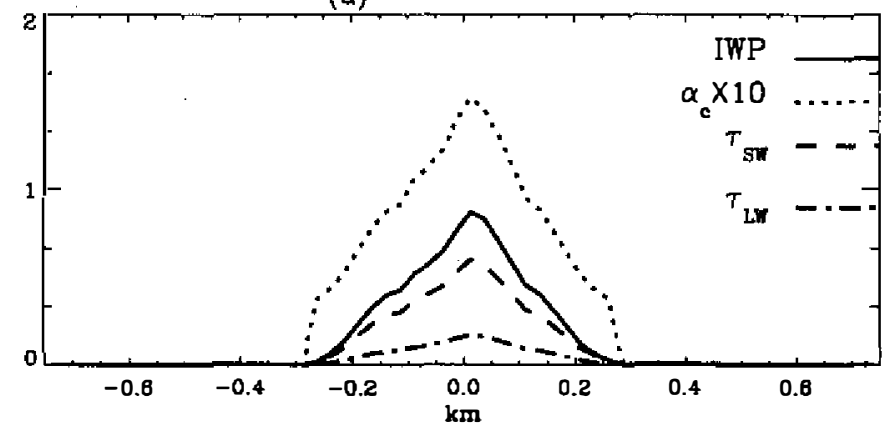

Fig. 4. Distributions of microphysical and optical properties of the dissipating contrail at $30 \mathrm{~min}$ simulation time: (a) ice water content, (b) ice particle number concentration, and (c) effective radius. Panel (d) shows the vertically integrated properties: ice water path (in $\mathrm{g} \mathrm{m}^{-2}$; solid curve), SW optical depth $\tau_{\text {sw }}$ (for wavelength between 0.3 to $0.7 \mu \mathrm{m}$, dashed curve), LW optical depth $\tau_{\mathrm{LW}}$ (for wavelength $10.75 \mu \mathrm{m}$, dashed-dotted curve), and cloud reflectivity $\alpha_{c}$ $(\times 10)$ at zenith angle $=$ $\cos ^{-1}(0.6)$ computed using IPA (dotted curve). 
(a) IWC (mg m-3)

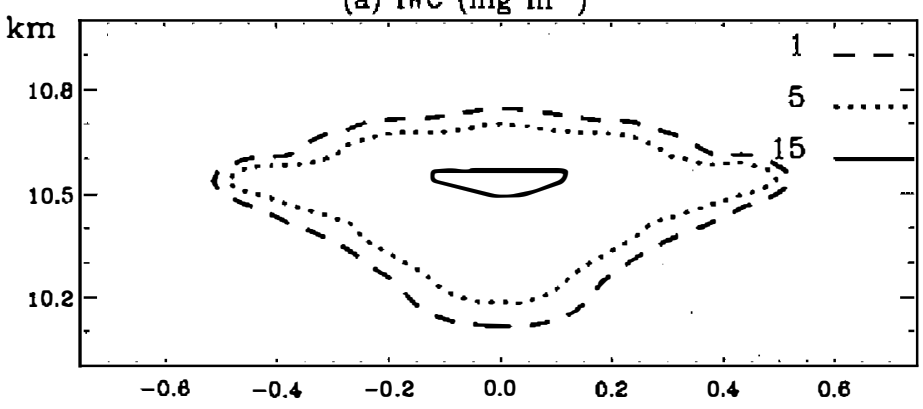

(b) $\mathrm{N}_{1}\left(\mathrm{~cm}^{-3}\right)$

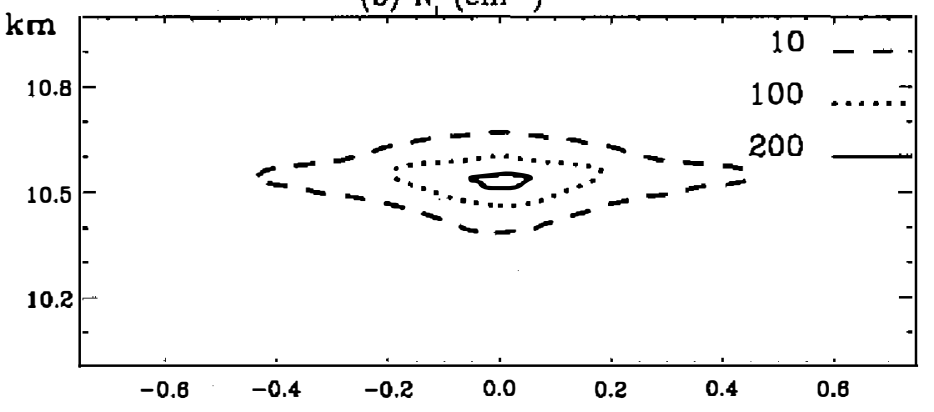

(c) $\mathrm{r} \cdot(\mu \mathrm{m})$

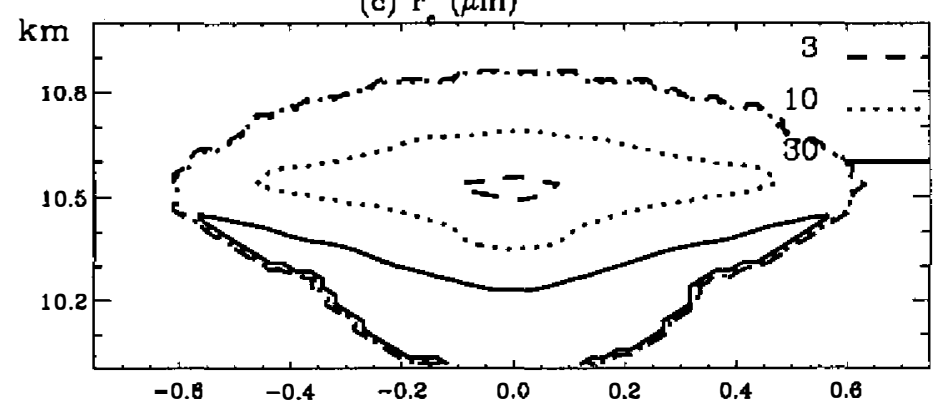

(d)

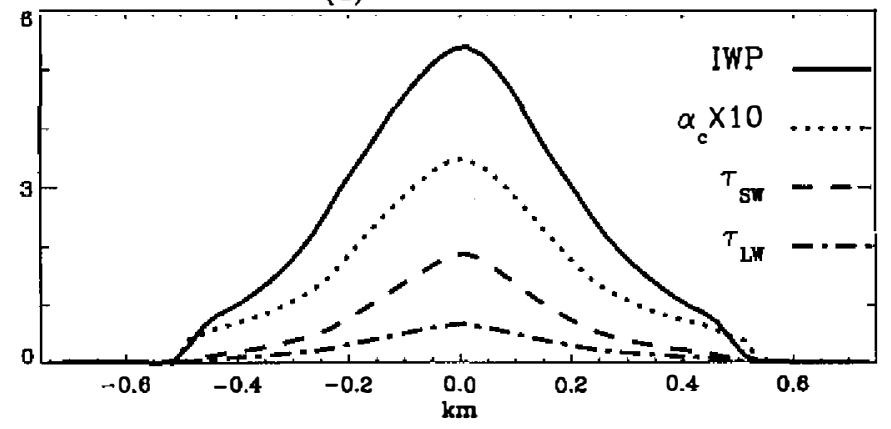

Fig. 5. Same as in Fig. 4, except for the persistent contrail. 
about 2 at the core of the persistent contrail, whereas only $1 / 3$ the value exists at the core of the dissipating contrail. Calculated with the MCM and IPA, the local cloud reflectivity can be over 0.4 and 0.1 at the center of the persistent and dissipating contrails, respectively. Therefore, one may expect the persistent contrails to be quite effective in reflecting sunlight and cooling the surface.

Ice particles in the dissipating contrail generally sublimate and decrease in size with time, but the details of this are less relevant and will not be discussed here. Let us focus on the size distributions of ice particles in the persistent contrail that shown in Fig. 6. At the cloud core, the size distribution is bimodal with a narrow primary mode peaked at about $2.3 \mu \mathrm{m}$ and a broad secondary mode at about $5 \mu \mathrm{m}$. At farther away from the core, the peak of the primary mode levels down and at the same time the secondary mode becomes broader. The appearance and subsequent broadening of the secondary mode is caused by the fast diffusional growth of particles at locations where high ice-supersaturation is present, namely, at the cloud edge, or in local updrafts. At the cloud edges, the primary mode totally disappears, so the distribution becomes unimodal and rather flat. At the lowest part of the cloud the particle distribution is unimodal but narrow with a peak at about $30 \mu \mathrm{m}$ (the dotted curve in Fig. 4b). These particles are actually the largest ice crystals that fall from the upper layers and continue to grow in the ice-supersaturated ambient air to form the fall streak. Note that the ice crystals in the fall streak must be large enough to overcome the lifting by the ascending air at the lower part of the contrail (see Fig. 3). Thus the distribution here has a lower cutoff at about $20 \mu \mathrm{m}$.

Without mixing, aggregation, sedimentation and particle nucleation, the size distributions of hydrometeors tend to become narrower with time in a supersaturated environment, which is
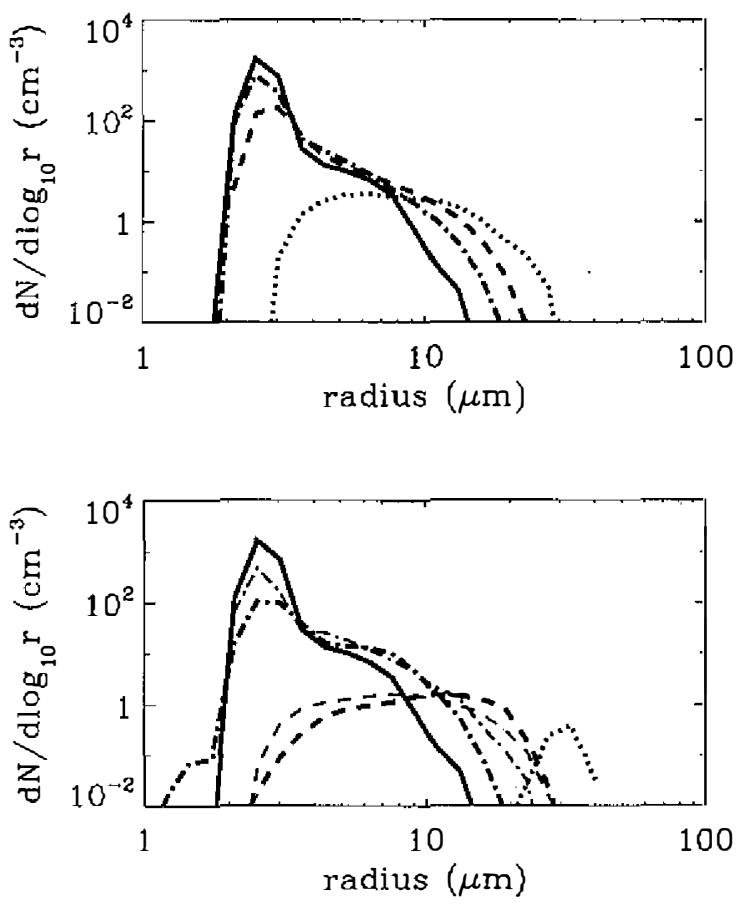

Fig. 6. Size distributions of ice particles at different locations in the persistent contrail at simulation time $30 \mathrm{~min}$. In the upper panel, the solid, dashed-dotted, dashed, and dotted curves denote those at sequential locations (150 $\mathrm{m}$ apart) from the cloud center to cloud edge in the horizontal direction. In the lower panel, the thin-dashed, thin dashdotted, solid, dash-dotted, dashed, and dotted curves indicate distributions at sequential locations (100 m apart) from cloud top to base. The solid curves in the two panels are from the same location, i.e., the center of the contrail. 
often demonstrated by typical parcel simulations (Warner 1969; Lee and Pruppacher 1977). In contrast, as shown in our simulations, growth takes place at the edge of the cloud but turbulent mixing tends to smoothen and broaden the spectrum (Fig. 7a). The larger crystals at the cloud edge are gradually entrained into the inner part of the contrail (Fig. 7b) and, at the same time, the smaller particles inside are also derained out to the cloud edge (Fig. 7a).

Next we will further discuss the radiation budget over a contrail according to the simulated radiative properties as given in Figs. 4 and 5 . Table 2 lists the domain-averaged reflectivity of the direct solar radiation for various incident solar azimuth and zenith angles. An azimuthal angle equal to $90^{\circ}$ indicates the incident light beam is parallel to the contrail, whereas $180^{\circ}$ indicates a perpendicular situation. We put down the domain-averaged reflectivity instead of the reflectivity of the cloudy region because the apparent cloudy area changes with the azimuth and zenith angles. So, the domain-averaged reflectivity gives us a sense of the cumulative effect of the whole contrail. In these two simulations, and within the 30 min simulation time, the average reflectivity is not sensitive to the azimuth angle. The reflectivity computed with the Monte Carlo method is somewhat smaller than that by IPA, but the differences are not significant in a statistical sense. This result agrees with the inference from the study by Schulz (1998).

Since the domain-averaged cloud reflectivity calculated with the two methods differ only slightly, we used the IPA method to estimate the SW radiative forcing (Table 3). Note that the listed quantities correspond to instantaneous values, unlike the daily mean (the SW radiative forcing is factored by the solar time over a 24-hour period) that is commonly quoted in the literature. From Table 3 one can see that the contrails are most likely to cool the atmosphere
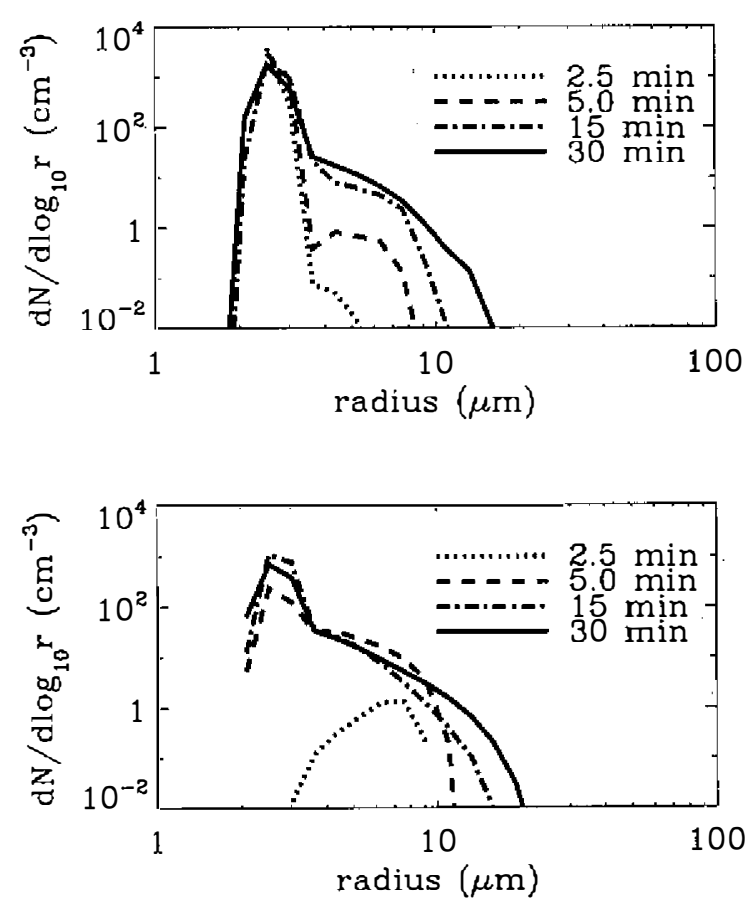

Fig. 7. Ice particle size distributions at different simulation times. Upper panel: near the cloud center at $\mathrm{x}=-0.01 \mathrm{~km}$ and $\mathrm{z}$ $=10.54 \mathrm{~km}$; Lower panel: near the cloud edge at $x=-0$. $19 \mathrm{~km}$ and $z=10.54 \mathrm{~km}$. 
Table 2. Domain-averaged reflectivity at various azimuth and zenith $\left(\mu_{0}\right)$ angles.

\begin{tabular}{|c|cc|cc|}
\hline Run 1 & \multicolumn{2}{|c|}{$10 \mathrm{~min}$} & \multicolumn{2}{c|}{$30 \mathrm{~min}$} \\
\hline azimuth angle & $\mu_{0}=0.6$ & $\mu_{0}=0.9$ & $\mu_{0}=0.6$ & $\mu_{0}=0.9$ \\
\hline $90^{\circ}$ & 0.035 & 0.016 & 0.022 & 0.010 \\
$135^{\circ}$ & 0.036 & 0.017 & 0.022 & 0.009 \\
$180^{\circ}$ & 0.035 & 0.016 & 0.021 & 0.009 \\
\hline IPA & 0.049 & 0.025 & 0.032 & 0.015 \\
\hline
\end{tabular}

\begin{tabular}{|c|cc|cc|}
\hline Run 2 & \multicolumn{2}{|c|}{$10 \mathrm{~min}$} & \multicolumn{2}{c|}{$30 \mathrm{~min}$} \\
\hline azimuth angle & $\mu_{0}=0.6$ & $\mu_{0}=0.9$ & $\mu_{0}=0.6$ & $\mu_{0}=0.9$ \\
\hline $90^{\circ}$ & 0.081 & 0.045 & 0.102 & 0.056 \\
$135^{\circ}$ & 0.081 & 0.045 & 0.104 & 0.057 \\
$180^{\circ}$ & 0.083 & 0.045 & 0.105 & 0.055 \\
\hline IPA & 0.087 & 0.048 & 0.112 & 0.061 \\
\hline
\end{tabular}

due to combined SW and LW radiative forcing, except under large surface albedo $\alpha_{\mathrm{s}}$ and small zenith angles. As discussed in the previous section, the ratio $Q_{\text {ext,SW}} / Q_{a b s, L W}$ increases sharply as the particle radius becomes smaller than $5 \mu \mathrm{m}$. One should expect that the threshold optical depth of contrails that contain mostly ice crystals with radius less than $10 \mu \mathrm{m}$ (cf. Fig. 6) are smaller than those in natural cirrus. However, this feature is not captured by many studies of contrail radiation budget because they adopted optical parameterization schemes designed for natural cirrus clouds. Our procedures did consider the variation in $Q_{e x, s w} / Q_{a b s, L W}$ and thus capture a highly peaked negative net radiative forcing over the contrail center.

\section{CONCLUSIONS}

A two-dimensional high-resolution cloud model is demonstrated to simulate reasonably well the evolution of contrails. Scenarios of a dissipating contrail and a persisting contrail are demons ated. As expected, the cloud coverage decreases with time in the dissipating scenario due to mixing with the ice-subsaturated ambient air, whereas the cloud coverage of persistent contrail increases with time in a saturated environment. At 30 min simulation time, ice particles are still concentrated in a region corresponding to the upper half of the initial contrail. In the persistent contrail scenario, ice crystals at the cloud edge grow by vapor diffusion as a result of mixing of ice-supersaturated ambient air. These large ice particles then further mix into the inner part of the contrail and cause the broadening of particle size distributions near 
Table 3. Domain-averaged cloud radiative forcing (in $\mathrm{W} \mathrm{m}^{-2}$ ) estimated with IPA when either $\mu_{0}=-0.6$ or -0.9 ; surface albedo either $\alpha_{s}=0.05$ or 0.3 .

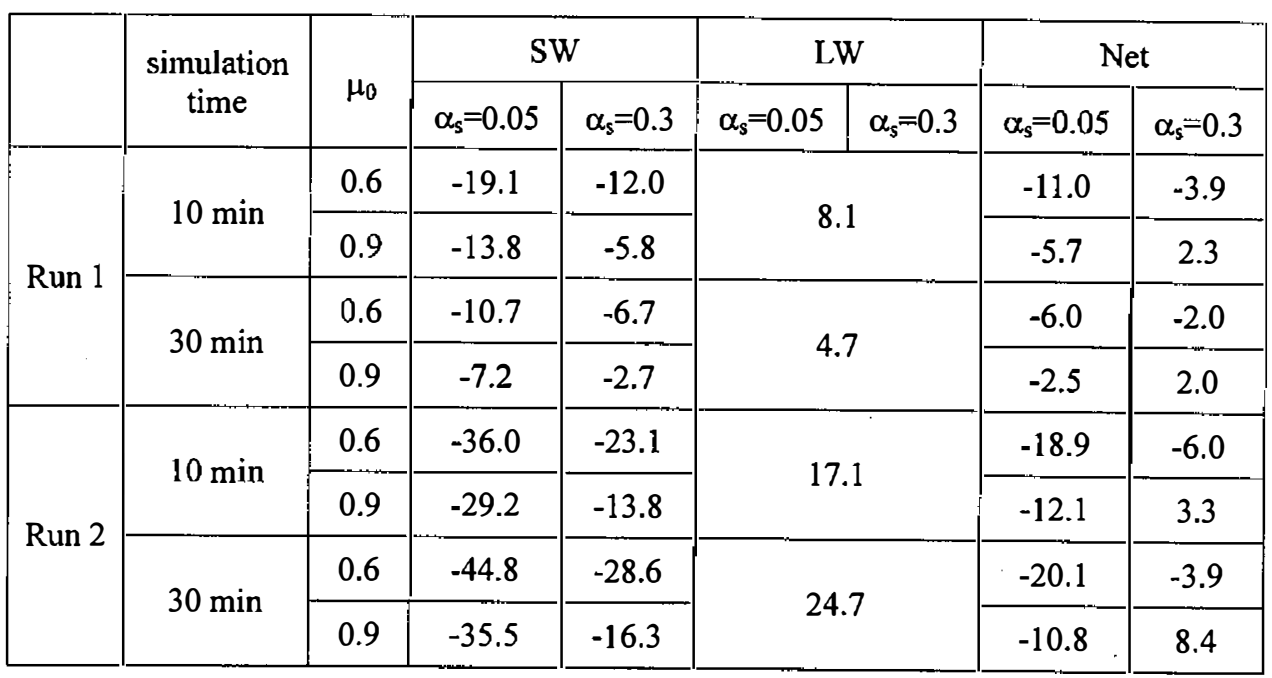

the contrail core. As a result, particle size distributions become bimodal in most of the cloudy region. Some of the largest ice crystals at the lower part of the contrails acquire significant fall speed and develope into a fall-streak at the bottom of the persistent contrail.

Cloud reflectivities under various zenith and azimuth angle conditions are computed using the Monte Carlo method and compared with those using the individual pixel approximation. We found that the IPA produces reasonable results in these two contrail scenarios. It is possible that the enhancement of cloud reflectivity due to cloud-side illumination as well as the reduction of cloud reflectivity caused by diffusion of radiation from cloud sides are both weak in these simulations. Our results also showed that the LW radiative forcing decreases with time in the dissipating scenario but increases with time in a persisting one. The SW radiative forcing, on the other hand, is sensitive to the surface albedo and the incident solar zenith angle. The magnitude of SW radiative forcing increases as surface albedo decreases. Therefore, contrails directly over the oceans (surface albdo $=0.05$ ) are more favorable for $\mathrm{SW}$ radiative cooling than contrails over a low-level cloud deck. A decrease in the incident solar zenith angle would result in decrease in the magnitude of SW radiative forcing. Generally speaking, the net radiative effect during daytime is a cooling of the surface for both contrail scenarios.

Such a negative cloud radiative effect seems to be quite different from those of many earlier studies, most of which showed a net warming effect. There are several reasons for that. First and foremost, the radiative forcing shown in this study is an instantaneous value at daytime with a given zenith angle, not a daily mean, which is usually adopted in contrail radiation budget studies. Secondly, this study grasps the actual variation of LW absorption efficiency of ice crystals as the particles become smaller than $10 \mu \mathrm{m}$. For contrails that contain mainly particles smaller than $10 \mu \mathrm{m}$ in radius, the ratios of extinction efficiency to absorption effi- 
ciency should be significantly greater than 2 as was used in this study. Our approach should provide better evaluation of the contrail effects on local-scale radiation budget.

The simulations we performed here are still quite idealized in terms of the atmospheric conditions, which were assumed to be motionless. As denoted by Chlond (1998), initializing the dynamic, thermodynamic, and cloud fields via the output of a jet-vortex-phase-resolving model (e.g., Gierens 1996) may overcome the drawback of the motionless atmosphere assumption. Also, we did not consider the vertical wind shear, which may cause the contrail to spread horizontally (Jensen et al. 1998), and evolve into a widespread cirrus deck. Further studies on the simulations of contrail-to-cirrus transition may improve our understanding of the role of these clouds in regional climate.

Acknowledgments This research was supported by the National Science Council under Grants NSC-87-2621-P-002-002, NSC-87-2811-M-002-0019 and NSC-88-2111-M-002-016.

\section{REFERENCES}

Ackerman, T. P., K. N. Liou, F. P. J. Valero, and L. Pfister, 1988: Heating rates in tropical anvils. J. Atmos. Sci., 45, 1606-1623.

Boehm, M. T., J. Verlinde, and T. P. Ackerman, 1999: On the maintenance of high tropical cirrus. J. Geophys. Res., 104, 24423-24433.

Boucher, O., 1999: Influence of air traffic on cirrus occurrence. Nature, 397, 30-31.

Changnon, S. A., 1981: Midwestern sunshine and temperature trends since 1901: possible evidence of jet contrail effects. J. Appl. Meteor., 20, 496-508.

Chen, J.-P., and D. Lamb, 1994: Simulation of cloud microphysical and chemical processes using a multicomponent framework. Part I: Description of the microphysical model. $J$. Atmos. Sci., 51, 2613-2630.

Chen, J.-P., G. M. McFarquhar, A. J. Heymsfield, and V. Ramanathan, 1997: A modeling and observational study of the detailed microphysical structure of mical cirrus anvils. $J$. Geophys. Res., 102, 6637-6653.

Chen, J.-P., W.-H. Lin, and R.-F. Lin, 2001: Estimation of contrail frequency and radiative effects over the Taiwan area. TAO, 12, 155-178.

Chlond, A., 1998: Large-eddy simulation of contrails. J. Atmos. Sci., 55, 796-819.

Chylek, P., and J. S. Dobbie, 1995: Radiative properties of finite inhomogeneous cirrus clouds: Monte Carlo simulations. J. Atmos. Sci., 52, 3512-3522.

Danielson, R. E., D. R. Moore, and H. C. van de Hulst, 1969: The transfer of visible radiation through clouds. J. Atmos. Sci., 26, 1078-1087.

Davies, R., 1978: The effect of finite geometry on the three-dimensional transfer of solar irradiance in clouds, J. Atmos. Sci., 35, 1712-1725.

Ebert, E. E., and J. A. Curry, 1992: A parameterization of ice cloud optical properties for climate model. J. Geophys. Res., 97, 3831-3836.

Fortuin, J. P. F., R. van Dorland, W. M. F. Wauben, and H. Kelder, 1995: Greenhouse effects of aircraft emissions as calculated by a radiative transfer model. Ann. Geophysicae, 13, 
413-418.

Frankel, D., K.-N. Liou, S.C. Ou, D. P. Wylie, and P. Menzel, 1997: Observations of cirrus cloud extent and their impacts to climate. Proceedings, Ninth Conference on Atmospheric Radiation, February 2-7, 1997, Long Beach , CA, Amer. Meteor. Soc., 414417.

Fu, Q., and K.-N. Liou, 1993: Parameterization of the radiative properties of cirrus clouds. $J$. Atmos. Sci., 50, 2008-2025.

Fu, Q., K. N. Liou, M. C. Cribb, T. P. Charlock, and A. Grossman, 1997: Multiple scattering parameterization in thermal infrared radiative transfer. J. Atmos. Sci., 54, 2799-2812.

Gierens, K. M., 1996: Numerical simulations of persistent contrails. J. Atmos. Sci., 53, 33333347.

Goodman, J., R. F. Pueschel, E. J. Jensen, S. Verman, G. V. Ferry, S. D. Howard, S. A. Kinne, and D. Baumgardner, 1998: Shape and size of contrails ice particles. Geophys. Res. Lett., 25, 1327-1330.

Hirsch, C., 1988: Numerical Computation of Internal and External Flows: Fundamentals of Numerical Discretization. John Wiley \& Son, Chichester, England, 538 pp.

Jensen, E. J., A. S. Ackerman, D. E. Stevens, O. B. Toon, and P. Minnis, 1998: Spreading and growth of contrails in a sheared environment. J. Geophys. Res., 103, 31557-31567.

Kinne, S., and K.-N. Liou, 1989: The effects of the nonsphericity and size distribution of ice crystals on the radiative properties of cirrus clouds. Atmos. Res., 24, 273-284.

Knollenberg, R. G., K. Kelly, and J. C. Wilson, 1993: Measurements of high number densities of ice crystals in the tops of tropical cumulonimbus. J. Geophys. Res., 98, 86398664.

Lee, I. Y., and H. R. Pruppacher, 1977: A comparative study on the growth of cloud drops by condensation using an air parcel model with and without entrainment. Pure Appl. Geophys., 115, 523-545.

Lilly, D. K., 1962: On the numerical simulation of a buoyant convection. Tellus, 14, 148-172.

Lilly, D. K., 1988: Cirrus outflow dynamics. J. Atmos. Sci., 45, 1594-1605.

Lin, R.-F., 1997: A numerical study of the evolution of nocturnal cirrus by a two-dimensional model with explicit microphysics. Ph.D. thesis, Pennsylvania State University, University Park, 199 pp.

Lin, R.-F., D. O'C. Starr, P. J. DeMott, R. Cotton, K. Sassen, and E. Jensen, 2000: Cirrus parcel model comparison project phase 1. Proceeding of the 13th International conference on clouds and precipitation, Reno, Navada.

Meerkotter, R., U. Schumann, D. R. Doelling, P. Minnis, T. Nakajima, and T. Tsushima, 1999: Radiative forcing by contrails. Ann. Geophysicae, 17, 1080-1094.

Meyers, M. P., P. J. DeMott, and W. R. Cotton, 1992: New primary ice-nucleation parameterizations in an explicit cloud model. J. Applied Meteorol., 31, 708-721.

Minnis, P., D. F. Young, D. P. Garber, L. Nguyen, W. L., Smith, Jr., and R. Palikonda, 1998: Transformation of contrails into cirrus during SUCCESS. Geophys. Res. Lett., 25, $1157-$ 1160 .

Minnis, P., U. Schumann, D. R. Doelhing, K. M. Gierens, and D. W. Fahey, 1999: Global 
distribution of contrail radiative forcing. Geophys. Res. Lett., 26, 1853-1856.

Schulz, J., 1998: On the effect of cloud inhomogeneity an area averaged radiative Properties of contrails. Geophys. Res. Lett., 25, 1427-1431.

Schumann, U., 1996: On conditions for contrail formation from aircraft exhausts. Meteorol. Z., 5, 4-23.

Seaver, W. L., and J. E. Lee, 1987: A statistical examination of sky cover changes in the contiguous United States. J. Climate Appl. Meteor., 26, 88-95.

Starr, D. O’C., and S. K. Cox, 1985: Cirrus clouds. Part I: A cirrus cloud model. J. Atmos. Sci., 42, 2663-2681.

Starr, D. O'C. and co-authors, 2000: Comparison of cirrus cloud models: A project of the GEWEX cloud system study (GCSS) working group on cirrus cloud systems. Proceeding of the 13th International Conference on Clouds and Precipitation, Reno, Nevada.

Stephens, G. L., S. Tsay, P. W. Stackhouse Jr., and P. J. Flatau, 1990: The relevance of the microphysical and radiative properties of cirrus clouds to climate and climate feedback. J. Atmos. Sci., 42, 2682-2694.

Toon, O. B., C. P. McKay, T. P. Ackerman, and K. Santhanam, 1989: Rapid calculation of radiative heating rates and photodissociation rates in inhomogeneous multiple scattering atmosphere. J. Geophys. Res., 94, D13, 16287-16301.

Walpole, R. E., and R. H. Myers, 1990: Probability and Statistics for Engineers and Scientists. Fourth Ed., Macmillan Publishing Company, New York, 765 pp.

Wang, W.-C, W. Gong, and J.-P. Chen, 2001: SUNYA regional model simulation of radiative forcing and climate responses due to contrails over regions around Taiwan. TAO, 12, 179-194.

Warner, J., 1969: The microstructure of cumulus cloud. Part I: General features of the droplet spectrum. J. Atmos. Sci., 26, 1049-1059.

Young, K. C., 1974: A numerical simulation of wintertime, orographic precipitation. Part I. Description of model microphysics and numerical techniques. J. Atmos. Sci., 31, 17351748. 